\title{
LES LOIS LES PLUS CONTRAIGNANTES DU MONDE
}

A côté des lois des hommes, écrites, spécifiques à chaque cité, il existe en Grèce des lois sacrées, non écrites, et communes à tous les Grecs. Ces lois, que l'on rencontre dans les mythes et la tragédie, plus contraignantes que les lois humaines, sont aussi en vigueur dans la société et nous observerons trois lois sacrées sous ce double point de vue : mythe et réel.

Le grec dispose de plusieurs mots pour parler des lois : tout d'abord du mot $\theta \varepsilon \sigma \mu$ ó qui a signifié "institution ou loi établie par les dieux, coutume antique », avant de désigner les lois faites par les hommes (les lois de Dracon par exemple à la fin du VII ${ }^{\circ}$ s. avt $\mathrm{JC}^{1}$ ) puis de disparaître au profit de vó $\mu$ os, loi (notamment loi écrite ${ }^{2}$ ). Il y a aussi le terme vó $\mu$ uov employé par l'Antigone de Sophocle qui parle de ó $\gamma \rho \alpha \pi \tau \alpha$ vó $\mu \mu \alpha$, «lois non écrites » (v. 454-455). «Je ne pensais pas que tes édits à toi fussent assez puissants pour permettre à un mortel de passer outre à d'autres lois, aux lois non écrites, inébranlables des dieux » (453-455, trad. P. Mazon), dit-elle à Créon après la mort de son frère Polynice et interdiction faite de l'enterrer sous peine de mort. Lois des dieux, donc sacrées, c'est-à-dire beaucoup plus graves à transgresser car rendant l'auteur de la transgression sacrilège. Ces lois appartiennent au premier des trois niveaux d'application de la justice, selon C. Baconicola, c'est-à-dire la «justice imposée directement par les dieux aux mortels ${ }^{3}$ », d'où leur caractère contraignant. On connaît par la littérature et les mythes plusieurs lois sacrées (ou lois non écrites) dont certaines ont trait aux relations avec des étrangers (loi d'hospitalité, loi envers les suppliants), mais dont plusieurs concernent les relations familiales, comme l'interdiction de faire couler le sang d'un membre de sa famille ou le devoir impératif pour les enfants de respecter leurs parents âgés (Eschyle, Les suppliantes, 707), de leur rendre les honneurs funèbres (Antigone et Ajax de Sophocle, Les suppliantes d'Euripide) ou encore celui pour les fils de venger leurs pères (Electre $)^{4}$.

Mais à lire l'ouvrage de Glotz sur La solidarité de la famille dans le droit criminel en Grèce $e^{5}$, dans lequel il étudie la solidarité des membres d'un génos (famille au sens large) en matière de justice et de lois, nous nous rendons assez vite compte que les œuvres littéraires et les mythes reflètent un état de la société. Cela est particulièrement clair lorsque nous avons la chance d'avoir à notre disposition des codes de lois ou des discours prononcés lors de procès, documents on ne peut plus précieux. Par exemple, parmi ses lois, Solon (au tout début du $\mathrm{VI}^{\circ}$ s. avt J.C.) inclut des lois communes à tous les génè et transmises par la tradition : la première est que «le fils est tenu de fournir à ses ascendants vieux ou pauvres les aliments et le gîte »; selon la deuxième «défense est faite au fils d'outrager ses ascendants dans leur personne ou leur honneur par voie de fait ou injure » et la troisième ordonne : «le fils est tenu de procurer à ses ascendants des funérailles et une sépulture honorables ${ }^{6} »$. Par ailleurs, la distinction entre lois écrites et non écrites existe encore en pleine époque classique, et un grand crédit est

\footnotetext{
${ }^{1}$ Aristote, Constitution d'Athènes, IV, 1.

2 Jacqueline de Romilly, La loi dans la pensée grecque, Les Belles Lettres, 2002, p. 17.

${ }^{3}$ Chara Baconicola, «La justice dans la tragédie grecque », dans Mythe et justice dans la pensée grecque, éd. Stamatios Tzitzis, Maria Protopapas-Marneli et Bjarne Melkevik, Presses de l'Université de Laval (Québec), 2009 , p. 175. Les deux autres niveaux sont la justice appliquée au plan interpersonnel et le troisième la justice appliquée au plan international.

${ }^{4}$ Plutarque, Isis et Osiris, 19, même idée : Horus estime que l'action la plus belle est « de venger son père et sa mère, quand ils ont été indignement traités », traduction M. Meunier.

${ }^{5}$ Gustave Glotz, La solidarité de la famille dans le droit criminel en Grèce, Paris, Albert Fontemoing éditeurs, 1904 , p. $14 ; 20$.

${ }^{6}$ G. Glotz, op. cité, p. 359-360.
} 
accordé à ces dernières. J. de Romilly nous dit la difficulté qu'il y a eu parfois à concilier les deux types de lois : celles qui étaient transmises par la coutume et étaient senties comme communes à tous les Grecs (p. 42), et celles que les législateurs mettaient en place : « la loi non écrite a [...] offert un recours à ceux que le nomos écrit laissait insatisfaits » (p. 46), et elle traduit «l'aspiration vers un bien et une justice qui compléteraient et dépasseraient les règles posées par le législateur » (p. 26).

On sait par deux sources différentes que Périclès faisait grand cas des lois non écrites. Ainsi, Lysias nous apprend que jadis il conseilla «d'appliquer aux impies non seulement les lois écrites [...] mais les lois non écrites ${ }^{7} \gg$; tandis que Thucydide, dans le discours qu'il lui attribue dans l'Histoire de la guerre du Péloponnèse, lui fait dire à propos des Athéniens :

« Nous obéissons toujours aux magistrats et aux lois et, parmi celles-

ci, surtout à celles qui assurent la défense des opprimés et qui, tout en

n'étant pas codifiées, impriment à celui qui les viole un mépris universel ${ }^{8} \gg$.

Voyons à présent d'un peu plus près trois de ces lois qui concernent les relations, droits et devoirs des membres de la famille entre eux :

\section{a) Nourrir et protéger ses vieux parents}

Chez Sophocle, à Ajax qui veut se suicider car il vient d'être humilié devant toute l'armée grecque avec la participation active de la déesse Athéna, sa compagne rappelle la règle : «Ecoute la voix de l'honneur. Il te défend d'abandonner ton père dans sa triste vieillesse, d'abandonner ta mère, ta mère chargée d'ans. [...] Aie pitié aussi de ton fils » (506510, trad. P. Mazon). On voit que le fils vient après les parents... Ajax ne sera cependant pas convaincu par son discours et ses arguments, et se suicidera quand même, mais il dictera à son fils le devoir de nourrir ses grands-parents à sa place (v. 568-570).

Les tragédies sont pleines de parents sans enfants, dont Egée est le plus célèbre, et qui expriment leur angoisse car comme le dit un personnage d'Euripide : « il n'y a rien de sûr pour un homme que ses enfants ${ }^{9} »$. Ailleurs, un autre de ses personnages nous apprend que «les fils restent au foyer pour honorer les dieux domestiques et les tombeaux ${ }^{10}$ ». Nombreux aussi sont ceux qui perdent leurs enfants et se lamentent : Pélée, après la mort de son petit-fils Pyrrhus (son fils Achille étant déjà mort) expose l'avenir qui l'attend : "Sans enfant, solitaire, sans voir la fin de mes maux, j'épuiserai ma vie de misères jusqu'à l'Hadès ${ }^{11}{ }$. Cadmos, à la mort de Penthée, son petit-fils, se plaint : «Toi que craignait la ville au point que nul n'osait faire affront au vieillard en voyant ton visage [...]. A présent, je serai chassé comme un infâme de ce palais ${ }^{12}{ }^{\prime}$.

En ce qui concerne la vie courante, nous trouvons chez Lysias l'idée que les Athéniens morts pendant la dictature des Trente ont été tués injustement à double titre : d'abord parce qu'ils étaient innocents et n'avaient fait «aucun tort à la cité », mais aussi parce qu'ils « laissaient des parents âgés qui attendaient de leurs enfants la subsistance de leur vieillesse, et une sépulture après leur mort ${ }^{13}$ ». Il rappelle aussi que « celui qui frappe son père naturel ou lui refuse des aliments, celui qui dépouille son père adoptif des biens qui lui appartiennent » mérite la peine de mort ${ }^{14}$.

\footnotetext{
${ }^{7}$ Contre Andocide, 10, trad. M. Bizos.

${ }^{8}$ Histoire de la guerre du Péloponnèse, II, 37, 3, trad. Jean Voilquin.

${ }^{9}$ Fg Oenée, 570, traduction H. Berguin et G. Duclos.

${ }^{10}$ Fg Danaé $\mathrm{n}^{\circ}$ 320, trad. H. Berguin et G. Duclos

${ }^{11}$ Euripide, Andromaque, 1217-18, trad. L. Méridier, Belles Lettres.

${ }^{12}$ Euripide, Les Bacchantes, 1310-1313, trad. H. Grégoire et J. Meunier, Belles Lettres.

${ }^{13}$ Contre Agoratos, 45, trad. L. Gernet.

${ }^{14}$ Ibid., 91, trad. L. Gernet
} 
Mais ce devoir des enfants est à double tranchant, et chez Euripide par exemple, Admète qui se fâche avec son père, (car celui-ci ne veut pas mourir à sa place, et c'est donc Alceste, la femme d'Admète qui sacrifiera sa vie pour son mari) lui dit : " procrée donc, sans perdre de temps, des fils qui nourriront ta vieillesse, mort te mettront au linceul et exposeront ton cadavre. Car ce n'est pas moi qui t'ensevelirai ${ }^{15} \gg$. Le fils a donc une arme contre son père s'il n'est pas content de lui, mais le risque est la malédiction et le châtiment inévitable. Nous connaissons tous les célèbres imprécations d'Edipe contre ses fils, qui aboutiront à leur mort mutuelle, car ils l'ont rejeté après la découverte de son «crime » et ne lui ont plus rendu les honneurs qui lui étaient dûs. Le père d'Admète est cependant plus clément et se contentera de tancer vertement son fils, lui faisant, pour se justifier, une remarque intéressante : je « t'ai engendré et nourri, mais mon devoir n'est pas de mourir à ta place. Les pères mourir pour les

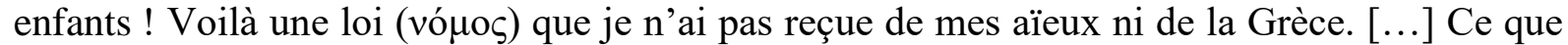
tu devais obtenir de nous, tu le possèdes ${ }^{16} »$. On voit donc que les droits et devoirs des uns et des autres étaient strictement codifiés.

\section{b) Venger son père}

Ce devoir impératif et absolu met en danger les enfants des victimes car les meurtriers, craignant leur vengeance, s'efforcent de les faire disparaitre. C'est la raison pour laquelle le fils d'Hector, Astyanax, a été jeté du haut des remparts de Troie (Eur. Troy. 719-725), les Grecs craignant que la ville ne revive dans le fils de son chef qui pourrait soulever une armée pour venger le mal fait à sa cité et à sa famille. C'est ce qui est en jeu après la guerre entre Argos et Thèbes à propos du conflit qui oppose les deux fils d'Edipe pour le pouvoir. La question de la sépulture des morts est assez brûlante pour qu'Euripide en fasse le sujet d'une de ses pièces, Les suppliantes. Le roi d'Argos ayant demandé l'aide de Thésée, celui-ci ironise en s'adressant au messager de Créon : «Que redoutez-vous [des morts] ? [...] Qu'ils n'engendrent dans les entrailles de la terre, des enfants dont la vengeance un jour vous atteindrait ? ${ }^{17}$ » Cette peur est en effet la conséquence du fait que dans le droit primitif, « qui a versé du sang doit du $\operatorname{sang}^{18}{ }$. Et à la fin de la pièce, c'est Athéna en personne qui s'adresse aux enfants des morts argiens en ces termes : "Aussitôt que vous serez des hommes, vous irez conquérir la ville de l'Ismène [Thèbes], vous vengerez la mort de vos pères défunts ${ }^{19}{ }$.

On trouve une situation différente mais liée à la même cause dans l'Héraklès d'Euripide. Lorsque Héraklès est aux Enfers, le maître de la ville veut tuer ses enfants car «il craint qu'un jour ces enfants, devenus grands, ne lui demandent compte du sang de leurs parents maternels ${ }^{20} \gg$ (qu'il a tués).

Dans le mythe des Atrides, Electre attend qu'Oreste, vengeur désigné de son père, accomplisse son devoir lorsqu'il atteindra l'âge de le faire. En effet, il a été mis à l'abri en exil car Egisthe voulait le tuer. De plus, Egisthe a empêché Electre de se marier : «dans la crainte qu'elle donnât à quelque grand un fils vengeur d'Agamemnon, Egisthe la gardait dans le palais, à toutes fiançailles refusant son accord ${ }^{21} »$. Ou alors, comme chez Euripide, il la marie, pour plus de sûreté, à un paysan :

Oreste : Mais Egisthe, pourquoi t'a-t-il fait cet outrage ?

\footnotetext{
${ }^{15}$ Alceste, 662-664, trad. L. Méridier.

${ }^{16}$ Ibid. 681-686.

${ }^{17}$ Euripide, Les suppliantes, 544-548, trad. L. Parmentier et H. Grégoire.

${ }^{18}$ G. Glotz, op. cité, p. 50.

${ }^{19}$ Euripide, Les suppliantes, 1213-1215, trad. L. Parmentier et H. Grégoire.

20 39-43, trad. L. Parmentier et H. Grégoire. Voir aussi v. 547.

${ }^{21}$ Euripide, Electre, 22-24, trad. L. Parmentier et H. Grégoire.
} 
Electre : Pour que, d'un tel époux, j'eusse des fils sans force.

Oreste : Il ne doit pas de toi naitre des fils vengeurs?

Electre : C'est son but. (266-269)

Ceci dit, malgré les torts d'Egisthe, il faut se souvenir qu'en tuant Agamemnon, il n'a fait que venger lui aussi, sur la personne du fils, le père qui avait humilié le sien et lui avait fait du mal. Atrée ayant tué les enfants de Thyeste (et les lui ayant de surcroît donnés à manger en ragoût), Egisthe était, dès avant sa naissance (en fait il a été conçu à cette seule fin), le vengeur de son père. La faute étant transmissible de père en fils, Egisthe a tué le fils (Agamemnon) pour le crime commis par son père (Atrée). C'est pourquoi, après avoir assassiné Agamemnon, il s'écrie que celui-ci a enfin payé : «les violences perfides d'un père ${ }^{22} \gg$.

Oreste quant à lui, se trouve prisonnier de deux lois non écrites et sacrées, et obéir à l'une l'oblige à désobéir à l'autre. En effet, d'un côté il a le devoir absolu de venger la mort de son père : « je te reçus des mains de l'un des tiens -ta propre sœur- et t'emportai, te nourris jusqu'à l'âge où tu es, pour que tu fusses un jour le vengeur de ce père ${ }^{23} »$, lui dit le pédagogue au début de la pièce de Sophocle. Chez Eschyle, c'est Apollon lui-même qui le pousse à la vengeance : l'oracle d'Apollon «m'annonçait des peines à glacer le sang de mon cœur, si je ne poursuivais les meurtriers d'un père par leurs propres voies et n'obéissais à son ordre : tuer qui a tué ${ }^{24} »$. Chez Euripide, Electre lui dit : « Ne défends pas ton père, et tu seras impie $[\ldots]$ qui te punira si ton père est invengé $?^{25} \gg$.

Mais de l'autre, il désobéit à une autre loi non écrite et sacrée qui interdit de faire couler le sang d'un membre de sa famille, en l'occurrence celui de sa mère Clytemnestre. Et après le meurtre, il pourra rétorquer à ceux qui l'accusent d'avoir tué sa mère : «si je suis un impie d'avoir tué ma mère, je porte un autre nom, celui d'homme pieux, car j'ai vengé mon père $^{26}{ }$. Il pourra même, dans Oreste, critiquer le refus de Ménélas (frère de son père) de le soutenir car «il est de notre sang et l'obligé de notre père ${ }^{27}{ }$. Chez Sophocle on ne nous dit rien d'une quelconque punition des dieux, et Eschyle trouve une solution juridique : par la création du premier tribunal qui l'absoudra. Dorénavant, ce seront des humains qui décideront de la culpabilité d'un homme, et les Euménides (déesses de la vengeance) ne pourront intervenir que si le tribunal décide de sa culpabilité. Mais pour qu'Oreste s'en sorte, il faudra trouver des arguments pour le moins douteux, imaginer que la mère n'est pas de la famille de son enfant, et ce sera le thème du plaidoyer d'Apollon dans Les Euménides d'Eschyle :

«Ce n'est pas la mère qui enfante celui qu'on nomme son enfant: elle n'est que la nourrice du germe en elle semé. Celui qui enfante, c'est l'homme qui la féconde ; elle, comme une étrangère, sauvegarde la jeune pousse $^{28} \gg$.

Si on observe la société athénienne, les enfants y avaient des devoirs impérieux envers leurs parents, et la sanction pour le non respect de ces règles était la colère des dieux, le mépris des hommes et la menace d'un talion ${ }^{29}$. Nous trouvons cette règle en pleine époque classique : chez Antiphon par exemple, l'accusateur tient la place du mort qui l'excite et le menace : «en ne vengeant pas la victime, nous susciterons contre nous un démon redoutable,

\footnotetext{
22 Eschyle, Agamemnon, 1582.

${ }^{23}$ Sophocle, Electre, 12-15, trad. P. Mazon.

${ }^{24}$ Les Choéphores, 270-275, trad. P. Mazon. Voir aussi Euménides, 465-467.

${ }^{25}$ Euripide, Electre, 976 et 978, trad. L. Parmentier et H. Grégoire.

${ }^{26}$ Euripide, Oreste, 546-547, trad. L. Méridier.

${ }^{27}$ Ibid., 244, trad. L. Méridier.

${ }^{28}$ Eschyle, Les Euménides, 658-661, trad. P. Mazon.

${ }^{29}$ G. Glotz, op. cité, p. 359.
} 
le fantôme du mort », dit-il. La vengeance est toujours un devoir sacré ${ }^{30}$. Platon, dans Les lois, parle des peines prévues à propos de meurtres involontaires. Si le meurtrier refuse de s'y soumettre, «le plus proche parent du défunt devra le poursuivre pour meurtre [...] Si le parent le plus proche néglige de poursuivre pour ce crime, la souillure se portera sur lui, pour ainsi dire, vu que la victime tournera vers lui ses réclamations ${ }^{31}{ }$.

Glotz nous dit également que dans le système de droit familial à époque archaïque, «plus encore qu'un droit, la vengeance du sang est un devoir» (p. 54) et un devoir « imprescriptible » (p. 55). Il remarque que ce devoir revient le plus souvent au fils, si jeune qu'il soit au moment de la mort de son père (p. 78), mais que le devoir de la femme, mère ou sœur, est de conserver la mémoire de ce devoir (p. 83).

On voit donc que le rôle que joue Electre dans le mythe et la tragédie, celui de la mémoire de la vengeance à accomplir, existe en fait dans la société, et même en pleine époque classique : c'est ainsi que nous lisons sous la plume de l'orateur Lysias à propos d'un homme qui va mourir: "ses recommandations suprêmes s'adressaient à sa femme qu'il croyait enceinte : s'il lui naissait un fils, elle devait lui dénoncer Agoratos comme meurtrier de son père et lui ordonner de poursuivre la vengeance du meurtre ${ }^{32} »$. Chez Isée, le père d'Astyphilos étant mort du fait de Thoudippos, père de Kléon, il enjoignit aux siens avant de mourir «d'interdire à jamais l'approche de son tombeau à tous les descendants de Thoudippos. [...] Astyphilos l'apprit [...] quand il était encore enfant et, dès qu'il eut l'âge de raison, il n'adressa jamais la parole à Kléon ; jusqu'à sa mort, il est resté persuadé qu'il

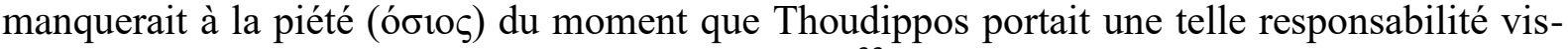
à-vis de son père, s'il échangeait un mot avec le fils ${ }^{33} »$.

Mais le droit et les mentalités ont évolué depuis l'époque archaïque jusqu'à l'époque classique, et les lois de Solon ont joué un rôle important dans l'émancipation de l'individu, comme le démontre Glotz dans son ouvrage déjà cité ${ }^{34}$. Les vieilles légendes rapportant des histoires de vengeance accomplie pour remplir son devoir ne sont plus perçues de la même façon qu'avant dans les tragédies athéniennes. En effet, la différence entre l'image d'Oreste dans l'épopée homérique et dans la tragédie classique a été maintes fois remarquée : jadis glorieux d'avoir vengé son père, il est pourchassé par les Erinyes chez Eschyle et Euripide. Ce changement d'image est sans doute lié à des croyances nouvelles, dont nous avons les premières traces écrites à la fin du $\mathrm{VIII}^{\circ}$ siècle, et qui concernent la souillure contractée par un crime sur un membre de sa famille ${ }^{35}$.

Avant de passer à la troisième loi examinée ici, il faut rappeler que l'être victime d'une violence peut également se venger seul : c'est ainsi que l'on peut comprendre le suicide d'Ajax ou d'Antigone. Le suicide, nous dit Glotz était la « vengeance des faibles » (p. 66), le «fantôme » du mort pouvant tourmenter son meurtrier. On se souvient que Créon, qui a condamné Antigone à mort, ne l'a pas fait tuer, mais l'a fait enfermer dans une grotte avec un peu de pain et d'eau. Façon habile et hypocrite de ne pas être responsable de la mort de celle qui était sa nièce, et ainsi de respecter la loi sacrée qui interdit de faire couler le sang d'un membre de sa famille. Mais le suicide de la jeune fille va court-circuiter ses précautions : il verra ainsi mourir successivement son fils Hémon et sa femme Eurydice.

\footnotetext{
${ }^{30}$ Antiphon, $3^{\circ}$ tétralogie, $\alpha, 4$, trad. L. Gernet.

${ }^{31}$ Lois, IX, 866, a-b, trad. A. Diès.

${ }^{32}$ Lysias, Contre Agoratos, 42, trad. M. Bizos. Voir aussi le paragraphe 41.

${ }^{33}$ La succession d'Astyphilos, 19-20, trad. P. Roussel.

${ }^{34}$ G. Glotz, op. cité, p. 325.

${ }^{35}$ Ibid., p. 231-233.
} 
Cette puissance d'outre-tombe de la victime en colère contre son meurtrier explique aussi bien la mutilation du cadavre d'Agamemnon dont Clytemnestre est accusée parfois ${ }^{36}$, que l'étrange échange entre Thésée et son fils à la fin d'Hippolyte. Thésée ayant été cause de la mort de son fils car il le croyait coupable de trahison, apprend qu'il est innocent et regrette, trop tard, ses imprécations, mais il a le temps de parler avec le mourant :

Thésée : Laisseras-tu mon âme avec une souillure ?

Hippolyte : Non, puisque je t'absous du crime de ma mort.

Thésée : Hé quoi ! Tu me renvoies absous du sang versé ?

Hippolyte : J'en atteste Artémis à l'arc irrésistible.

Thésée : Bien aimé, que tu es généreux pour ton père $!^{37}$

\section{c) Enterrer les morts}

Dans le mythe d'Antigone, nous voyons la jeune fille écartelée entre les lois humaines d'une part, les lois divines, non écrites de l'autre, ce qui la conduira à la mort ; écartelée entre son désir de se marier et d'avoir des enfants, en un mot de vivre, et le devoir absolu auquel elle doit obéir, qui est de donner une sépulture à son frère :

« [Créon] m'a saisie, il m'emmène et je n'aurai connu ni le lit nuptial ni le chant d'hyménée ; je n'aurai pas eu, comme une autre, un mari, des enfants grandissant sous mes yeux ; mais, sans égards, abandonnée des miens, misérablement, je descends, vivante, au séjour souterrain des morts ! Quel droit divin pourtant ai-je offensé ? ${ }^{38}{ }$

L'obligation d'enterrer les morts (et pas seulement les parents) est évidemment en vigueur dans la société grecque, et Lysias nous apprend que les parents âgés attendent de leurs enfants « la subsistance de leur vieillesse et une sépulture après leur mort ${ }^{39}$ », comme on l'a déjà vu. Isocrate, dans un discours, reprend l'exemple de la guerre contre Thèbes et du refus de Créon de rendre leurs morts aux Argiens (dont nous avons dit un mot un peu plus haut). Il parle à ce propos d'un "usage ancien, une loi ancestrale, que l'ensemble des hommes n'a cessé de pratiquer, non parce que la nature humaine l'a établie, mais parce qu'une puissance divine l'a imposée ${ }^{40} \gg$. Comme l'avait fait Antigone en s'adressant à Créon, il reprend l'idée de la loi des dieux, non écrite, à laquelle on ne peut pas se soustraire et qui est supérieure à celle, éphémère, des hommes. D'ailleurs il ajoute un détail d'importance, précisant qu'il s'agit d'une loi pratiquée par "l'ensemble des hommes », donc d'une loi commune à tous les Grecs et non celle d'un état particulier. C'est en effet une des caractéristiques des lois non écrites et sacrées que d'être panhelléniques.

Par ailleurs, la solidarité familiale qui oblige le fils à donner sépulture et à venger le père, a pour conséquence qu'il soit frappé en même temps que son père si celui-ci est condamné ${ }^{41}$. Cependant, nous assistons à une évolution des pratiques, surtout à Athènes et à un changement de taille, celui des progrès de l'individu, qui va faire lire les vieilles légendes différemment et amener à désolidariser les enfants de leurs parents lors de châtiments. Les sophistes, Protagoras notamment, joueront un rôle dans ce tournant. Ce sont eux qui vont contribuer à dégager le droit pénal de la théologie. Car nous dit Glotz, « quelle est cette justice qui punit les fils et les pères avec une rigueur imprescriptible ? C'est la justice divine. [...]

\footnotetext{
${ }^{36}$ Eschyle, Les Choéphores, 439 ; Sophocle, Electre, 442-446.

${ }^{37}$ Euripide, Hippolyte, 1448-1455, trad. L. Méridier.

${ }^{38}$ Sophocle, Antigone, v. 916-921, trad. P. Mazon.

${ }^{39}$ Contre Agoratos, 45, trad. M. Bizos.

${ }^{40}$ Isocrate, Panathénä̈que, 169, trad. G. Mathieu et E. Brémond. Mais G. Glotz, op. cité, p. 460 signale que les tyrans, les traîtres et les sacrilèges étaient privés de sépulture selon une loi admise par tous les Grecs.

${ }^{41}$ G. Glotz, op. cité, p. 458 ; 467 ; 468.
} 
Une pareille justice ne convient pas à l'homme. Il la subit, il constate les coups qu'elle frappe ; mais pour qu'il l'approuve et soit tenté de l'imiter, il faut qu'à chaque victime qu'elle fait il reconnaisse une faute personnelle » (p. 410). Ainsi, lorsque le fils d'Alcibiade aura un procès, il sera question des crimes de son père. Nous avons deux témoignages. Lysias, dans son discours Contre Alcibiade, rappelle que «tout enfant [...] cet homme [le fils d'Alcibiade] a failli être livré aux Onze à cause des crimes paternels ${ }^{42} »$. De son côté, Isocrate, faisant parler l'accusé dans son discours Sur l'attelage, lui fait dire : "car certes, on ne me châtiera pas pour ce qu'a fait mon père ${ }^{43} »$. Platon va dans le même sens dans Les lois lorsqu'il pose que « les hontes et les châtiments du père ne suivront jamais les enfants, sauf si père, grandpère et père du grand-père ont été, l'un après l'autre, condamnés à mort ${ }^{44} »$. Et Glotz note que dès 410/411 on ne voit plus à Athènes la peine capitale appliquée aux enfants des traîtres (p. 468).

Dans la tragédie on entendra un (Edipe se plaindre de l'injustice de son sort qui lui a imposé des crimes qui ne sont pas les siens. Lorsqu'il arrive à Colone (faubourg d'Athènes) il dit aux Athéniens qui hésitent à l'accueillir : «Mon nom seul vous fait peur. Car ce n'est certes pas ma personne ou mes actes. Mes actes, je les ai subis et non commis, s'il m'est permis d'évoquer à mon tour ceux de mes père et mère. [...] C'est sans rien savoir que j'en suis venu où j'en suis venu; tandis qu'ils savaient, eux, ceux par qui j'ai souffert et qui voulaient ma mort ! ${ }^{45} »$.

\section{Conclusion}

On a pu voir au cours de ce parcours trop superficiel, que les lois non écrites ont un caractère contraignant extrêmement puissant, et peuvent conduire à des situations extrêmes. La tragédie grecque nous montre plusieurs cas où des enfants sont pris entre la nécessité d'y obéir, et leurs débats internes, les déchirements que produisent le respect ou le non-respect de ces lois, comme Antigone et Oreste qui sont les plus célèbres, mais pas les seuls. Si ces lois ne sont jamais remises en question dans les textes tragiques ou poétiques grecs, on nous y montre cependant que leur stricte application, loin d'apporter bonheur et sérénité du devoir accompli, peut conduire à la folie (Oreste) ou à la mort (Antigone).

Par ailleurs, Glotz a montré l'évolution de la loi familiale à la loi de la cité qui rognera peu à peu les prérogatives de la famille, et Romilly a étudié la crise de la notion de loi à l'époque classique. De son côté, Castoriadis attire l'attention sur un chant du chœur de l'Antigone de Sophocle, et l'on pourrait conclure là-dessus : «ce chant du chœur finit, dit-il, sur l'éloge de l'homme capable de «tisser ensemble» les lois de la cité et la justice des dieux ${ }^{46} »$. La traduction de P. Mazon (Budé) dit : « qu'il [l'homme] fasse donc dans ce savoir une part aux lois de sa ville et à la justice des dieux, à laquelle il a juré foi $!^{47} \gg$. Et une autre traduction (R. Pignarre) : «sur la justice éternelle il greffe les lois de la terre ${ }^{48}$ ». En fait, le verbe grec $\pi \alpha \rho \varepsilon i ́ p \omega$ signifie d'abord insérer, introduire, mais aussi mélanger. C'est cette idée de «mélange » que la traduction de Castoriadis et celle de Pignarre essaient de rendre par les images du tissage et de la greffe, images bien plus fructueuses que celle de Mazon, car elles montrent qu'il y a une voie à trouver, viable ou supportable pour l'homme, et qui est celle de la prise en compte aussi bien du divin que de l'humain. L'un ou l'autre seul aboutit à des abus de pouvoir injustes (loi de Créon) ou à des situations inextricables (Antigone, Oreste). Belle

\footnotetext{
${ }^{42}$ Contre Alcibiade, 17, trad. M. Bizos. Les Onze étaient chargés de l'exécution des condamnés à mort.

${ }^{43}$ Sur l'attelage, 44, trad. G. Mathieu et E. Brémond.

${ }^{44}$ Lois, IX, 856, c-d, trad. A. Diès.

${ }^{45}$ CEdipe à Colone, 265-272, Trad. P. Mazon. C'est la dernière pièce de Sophocle, représentée après sa mort, en 401.

${ }^{46}$ C. Castoriadis, La cité et ses lois, Ce qui fait la Grèce, 2 , Seuil, 2008, p. 142-143.

47 368-369, trad. P. Mazon.

48 Trad. R. Pignarre, GF.
} 
leçon de sagesse que nous donne Sophocle, mais Eschyle avait donné un exemple de ce même tressage dans lequel l'humain peut trouver sa juste place, avec la création des tribunaux qui conservaient cependant aux déesses leurs prérogatives en cas de culpabilité reconnue par les humains, comme nous l'avons vu dans Les Euménides. On a l'impression que la société athénienne a essayé de corriger et de limiter, par ses lois humaines et écrites, celles de Solon d'abord, puis celles d'Euclide en 403, les dégâts occasionnés par certaines lois sacrées, dont le souvenir restait très vivace dans la société et les tragédies ${ }^{49}$.

\author{
Mireille Brémond \\ IEFEE \\ Université d'Aix-Marseille
}

${ }^{49}$ G. Glotz, op. cité, p. 607. 